\title{
The utility of the brain trauma evidence to inform paramedic rapid sequence intubation in out-of-hospital stroke
}

\author{
Pieter Francsois Fouche ${ }^{1 *}$ (D) Paul Andrew Jennings ${ }^{2}$, Malcolm Boyle ${ }^{3}$, Stephen Bernard ${ }^{4}$ and Karen Smith ${ }^{1,4,5}$
}

\begin{abstract}
Background: Rapid sequence intubation (RSI) is used to secure the airway of stroke patients. Randomized controlled trial evidence exists to support the use of paramedic RSI for traumatic brain injury (TBI), but cannot necessarily be applied to stroke RSI because of differences between the stroke and TBI patient. To understand if the TBI evidence can be used for stroke RSI, we analysed a retrospective cohort of TBI and strokes to compare how survival is impacted differently by RSI when comparing strokes and TBI.

Methods: This study was a retrospective analysis of 10 years of in-hospital and out-of-hospital data for all stroke and TBI patients attended by Ambulance Victoria, Australia. Logistic regression predicted the survival for ischemic and haemorrhagic strokes as well as TBI. The constituents of RSI, such a medications, intubation success and time intervals were analysed against survival using interactions to asses if RSI impacts survival differently for strokes compared to TBI.
\end{abstract}

Results: This analysis found significant interactions in the RSI-only group for age, number of intubation attempts, atropine, fentanyl, pulse rate and perhaps scene time and time- to-RSI. Such interactions imply that RSI impact survival differently for TBI versus strokes. Additionally, no significant difference in survival for TBI was found, with a $-0.7 \%$ lesser survival for RSI compared to no-RSI; OR 0.86 ( $95 \% \mathrm{Cl} 0.67$ to $1.11 ; p=0.25$ ). Survival for haemorrhagic stroke was $-14.1 \%$ less for RSI versus no-RSI; OR 0.44 ( $95 \%$ Cl 0.33 to $0.58 ; p=0.01)$ and was $-4.3 \%$; OR 0.67 (95\% Cl 0.49 to $0.91 ; p=0.01$ ) lesser for ischemic strokes.

Conclusions: Rapid sequence intubation and related factors interact with stroke and TBI, which suggests that RSI effects stroke survival in a different way from TBI. If RSI impact survival differently for strokes compared to TBI, then perhaps the TBI evidence cannot be used for stroke RSI.

Keywords: Traumatic brain injury, Stroke, rapid sequence intubation, Paramedic

\section{Background}

Strokes account for $10 \%$ of deaths worldwide [1]. Rapid Sequence intubation (RSI) is used in the emergency setting to improve survival in strokes, with perhaps 6 to $79 \%$ of strokes receiving intubation, depending on the stroke type [2]. Rapid sequence intubation is used to secure the airway using sedative and paralytic drugs to facilitate endotracheal intubation [2]. Strokes form 37\% of RSI for non-traumatic brain pathologies undertaken by

\footnotetext{
* Correspondence: Pieter.Fouche@monash.edu

${ }^{1}$ Department of Community Emergency Health and Paramedic Practice,

Monash University, Melbourne, Australia

Full list of author information is available at the end of the article
}

paramedics in Victoria, Australia [3]. Despite the notinfrequent use of RSI in unconscious out-of-hospital acquired brain injuries, no high quality evidence exists to support the use of RSI for strokes [2-4]. A randomized controlled trial of RSI in traumatic brain injury (TBI) exist [5], but the evidence from this trial cannot necessarily be applied to stroke RSI due to differences between the stroke and TBI patient [2-4].

Lower RSI survival for strokes compared to TBI suggests that the evidence from TBI might not be applicable to RSI in stroke [4]. It is important to investigate if brain trauma RSI evidence is transferable to strokes. That is, it would be vital to understand the RSI components that

(c) The Author(s). 2020 Open Access This article is distributed under the terms of the Creative Commons Attribution 4.0 International License (http://creativecommons.org/licenses/by/4.0/), which permits unrestricted use, distribution, and 


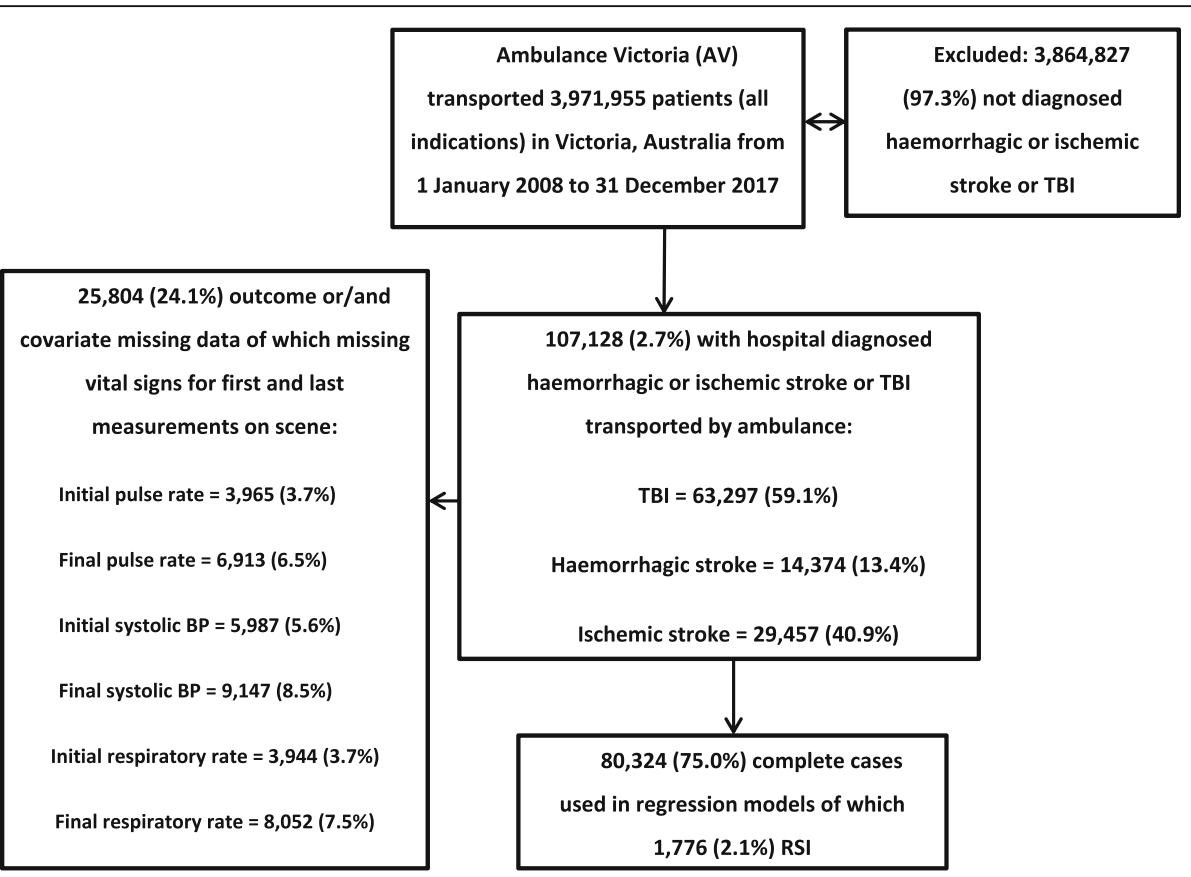

Fig. 1 Patient selection for a cohort of RSI in stroke and TBI

cause the survival difference of TBI compared to strokes. This study aims to find the constituents of RSI that could cause different survival between TBI and strokes. If any component of RSI impacts survival differently for TBI compared to strokes, it would follow that RSI itself has a different effect on these two pathologies. If RSI causes dissimilar survival for these two illnesses, then this would mean that the RSI TBI evidence-base cannot be transferred to strokes. Any such differences would imply that a stroke RSI trial is needed.

\section{Methods}

\section{Study setting and data sources}

Victoria has 6.3 million residents serviced by a two-tier emergency medical service. Mobile Intensive Care Paramedics are authorized to provide RSI to patients that have a Glasgow Coma Scale of less than 10 using suxamethonium as the primary paralytic, with pancuronium used to maintain paralysis [6]. Midazolam, morphine/ midazolam infusions, atropine, ketamine and fentanyl were available to aid RSI [6]. This study analysed data from 131 hospitals and clinics in Victoria, Australia for the 10 year period 1 January 2008 to 31 December 2017. Monash University Human Research Ethics Committee provided ethics approval (ref. no. 8618).

\section{Selection of cohort}

This study included all patients of any age that were treated and transported by Ambulance Victoria, with a hospital diagnosis of TBI or stroke. We excluded transient ischemic attack and strokes that could not be classified as either haemorrhagic or ischemic. Instances of stroke and TBI were identified by the Australian modification of ICD10 codes: S06 (Intracranial injury), I60 (subarachnoid haemorrhage), I61 (intracerebral haemorrhage), I62.9 (intracranial haemorrhage [non-traumatic], unspecified) and I63 (cerebral infarction). We initially selected all hospital records of patients with TBI and stroke codes, irrespective of ambulance transportation. These TBI and stroke in-hospital records were then linked to the Ambulance Victoria out-of-hospital records to select those that had a stroke or TBI and were transported by ambulance [4].

\section{Predictors and outcomes}

The primary outcome was survival to hospital discharge. Potential predictors include demographic, treatment, baseline observations ("vital signs") and scene/transport time intervals. Illness severity and comorbidity are important confounders in strokes and TBI, and we adjusted for illness severity using Glasgow Coma Scale. Whilst Glasgow Coma Scale was not specifically designed as a severity score for TBI and strokes, it could be similarly predictive of inhospital survival as NIHSS for strokes [7], and has strong prognostic value in TBI [8]. We adjusted for comorbidity by calculating the Walraven-Elixhauser comorbidity score [9]. No adjustment for any in-hospital interventions were done to avoid conditioning on a mediator variable [10]. 
Table 1 Patient, demographic, prognostic and in-hospital factors in in a cohort of 80,324 strokes and traumatic brain injury

\begin{tabular}{|c|c|c|c|}
\hline \multirow[t]{2}{*}{ Characteristic } & \multicolumn{3}{|l|}{ Patients, No. (\%) } \\
\hline & Total $(n=80,324)$ & $\mathrm{RSI}(n=1776)$ & No-RSI $(n=78,548)$ \\
\hline \multicolumn{4}{|l|}{ Demographic } \\
\hline Age, mean (SD), years & $61.6(22.5)$ & $52.2(22.5)$ & $61.8(24.2)$ \\
\hline \multicolumn{4}{|l|}{ Sex } \\
\hline Male & $46,726(58.1)$ & $1125(63.3)$ & $45,601(58.1)$ \\
\hline Female & $33,598(41.8)$ & $651(36.7)$ & $32,947(41.6)$ \\
\hline \multicolumn{4}{|l|}{ Illness } \\
\hline Haemorrhagic stroke & $11,133(13.9)$ & $531(29.9)$ & $10,602(13.5)$ \\
\hline Ischemic stroke & $26,754(33.3)$ & $242(13.6)$ & $26,512(33.8)$ \\
\hline Traumatic brain injury & $42,437(52.9)$ & $1003(56.5)$ & $41,434(52.8)$ \\
\hline \multicolumn{4}{|l|}{ Illness severity/comorbidity, mean (SD) } \\
\hline Elixhauser comorbidity score ${ }^{a}$ & $17.2(7.0)$ & $17.3(6.6)$ & $17.2(7.0)$ \\
\hline Initial Glasgow Coma Scale & $13.5(2.8)$ & $6.4(3.6)$ & $13.7(2.5)$ \\
\hline \multicolumn{4}{|l|}{ Observations, mean (SD) } \\
\hline Initial pulse rate & $85.4(19.5)$ & $92.9(28.8)$ & $85.2(19.2)$ \\
\hline Final pulse rate & $82.6(18.0)$ & $101.5(22.4)$ & $82.1(17.6)$ \\
\hline Initial systolic blood pressure & $140.6(31.5)$ & $145.7(41.8)$ & $140.5(31.2)$ \\
\hline Final systolic blood pressure & $139.3(28.5)$ & $136.4(29.7)$ & $139.3(28.5)$ \\
\hline Initial respiratory rate & $17.7(4.6)$ & $18.3(8.0)$ & $17.7(4.5)$ \\
\hline Final respiratory rate & $16.9(4.0)$ & $12.2(7.5)$ & $17.0(3.8)$ \\
\hline Initial $\mathrm{SPO}_{2}$ & $96.1(5.1)$ & $94.1(9.6)$ & $96.1(4.9)$ \\
\hline Final $\mathrm{SPO}_{2}$ & $97.2(3.4)$ & $98.5(4.6)$ & $97.2(3.3)$ \\
\hline \multicolumn{4}{|l|}{ Ambulance time intervals, minutes mean (SD) } \\
\hline Response time & $21.1(23.1)$ & $19.2(18.9)$ & $21.2(23.2)$ \\
\hline Scene time & $24.3(99.9)$ & $60.7(35.0)$ & $23.4(100.7)$ \\
\hline Transport time & $25.6(21.0)$ & $31.5(23.5)$ & $25.5(20.9)$ \\
\hline \multicolumn{4}{|l|}{ Hospital } \\
\hline Time in intensive care unit, mean (SD), hours & $125.2(161.3)$ & $156.9(172.2)$ & $119.4(158.5)$ \\
\hline Mechanical ventilation in intensive care unit, mean (SD), hours & $106.8(148.9)$ & $117.9(144.6)$ & $103.8(150.0)$ \\
\hline Hospital length of stay, mean (SD), days & $6.8(10.2)$ & $13.3(20.5)$ & $6.6(9.8)$ \\
\hline Emergency department length of stay mean (SD), minutes & $426.1(318.7)$ & $272.2(214.8)$ & $430.2(320.0)$ \\
\hline \multicolumn{4}{|l|}{ Year } \\
\hline 2008 & $6497(8.1)$ & $148(8.3)$ & $6349(8.1)$ \\
\hline 2009 & $7435(9.3)$ & $142(8.0)$ & $7293(9.3)$ \\
\hline 2010 & $7834(9.8)$ & $167(9.4)$ & $7667(9.8)$ \\
\hline 2011 & $8614(10.7)$ & $215(12.1)$ & $8399(10.7)$ \\
\hline 2012 & $8293(10.3)$ & $205(11.5)$ & $8088(10.3)$ \\
\hline 2013 & $7962(9.9)$ & $194(10.9)$ & $7768(9.9)$ \\
\hline 2014 & $6416(8.0)$ & $123(6.9)$ & $6293(8.0)$ \\
\hline 2015 & 8581 (10.7) & $164(9.2)$ & 8417 (10.7) \\
\hline 2016 & 9101 (11.3) & $221(12.4)$ & 8880 (11.3) \\
\hline 2017 & 9591 (11.9) & $197(11.1)$ & 9394 (12.0) \\
\hline
\end{tabular}


Table 2 Comparison of changes in vital signs in a cohort of 80,324 strokes and traumatic brain injury

\begin{tabular}{|c|c|c|c|}
\hline Change in vital sign ${ }^{\mathrm{a}}$ & RSI (mean; 95\% Cl) & No-RSI (mean; 95\% Cl) & Difference (95\% Cl; P) \\
\hline \multicolumn{4}{|l|}{ Haemorrhagic strokes } \\
\hline Systolic blood pressure $(\mathrm{mmHg})$ & $-15.8(-17.8$ to -13.9$)$ & $-2.0(-2.4$ to -1.6$)$ & $-13.8(-15.8$ to $-11.8 ; p<0.001$ \\
\hline $\mathrm{SPO}_{2}(\%)$ & 2.0 (1.4 to 2.5$)$ & 1.8 (1.7 to 2.0$)$ & $0.1(-0.5$ to $0.7 ; p=0.72)$ \\
\hline Respiratory rate (per minute) & $-5.8(-6.1$ to -5.5$)$ & $-0.4(-0.5$ to -0.4$)$ & $-5.4(-6.1$ to $-5.5 ; p<0.001)$ \\
\hline Glasgow Coma Scale (unit) & $-3.5(-3.6$ to -3.3$)$ & $-0.08(-0.1$ to -0.04$)$ & $-3.4(-3.5$ to $-3.2 ; p<0.001)$ \\
\hline Pulse (per minute) & $13.2(11.9$ to 14.3$)$ & $-1.7(-1.9$ to -1.4$)$ & $14.8(13.6$ to $16.1 ; p<0.001)$ \\
\hline \multicolumn{4}{|l|}{ Ischemic strokes } \\
\hline Systolic blood pressure (mmHg) & $-9.8(-12.1$ to -1.1$)$ & $-1.3(-1.5$ to -1.1$)$ & $-8.5(-10.8$ to $-6.2 ; p<0.001)$ \\
\hline $\mathrm{SPO}_{2}(\%)$ & 4.2 (3.6 to 4.8$)$ & $1.2(1.1$ to 1.3$)$ & $3.0(2.3$ to $3.6 ; p<0.001)$ \\
\hline Respiratory rate (per minute) & $-6.8(-7.1$ to -6.5$)$ & $-0.5(-0.48$ to -0.43$)$ & $-6.4(-6.7$ to $-6.1 ; p<0.001)$ \\
\hline Glasgow Coma Scale (unit) & $-3.9(-4.1$ to -3.8$)$ & 0.1 (0.1 to 0.14$)$ & $-4.0(-4.2$ to $-3.9 ; p<0.001)$ \\
\hline Pulse (per minute) & 4.7 (3.3 to 6.0$)$ & $-1.5(-1.6$ to -1.4$)$ & $6.2(4.8$ to $7.6 ; p<0.001)$ \\
\hline \multicolumn{4}{|l|}{ Traumatic brain injuries } \\
\hline Systolic blood pressure $(\mathrm{mmHg})$ & $-2.6(-3.6$ to -1.5$)$ & $-1.3(-1.4$ to -1.1$)$ & $-1.3(-2.4$ to $-0.2 ; p=0.02)$ \\
\hline $\mathrm{SPO}_{2}(\%)$ & 2.2 (1.8 to 2.5$)$ & $1.0(0.9$ to 1.1$)$ & $1.2(0.8$ to $1.5 ; p<0.001)$ \\
\hline Respiratory rate (per minute) & $-6.1(-6.3$ to -5.9$)$ & $-0.9(-0.9$ to -0.85$)$ & $-5.2(-5.4$ to $-5.0 ; p<0.001)$ \\
\hline Glasgow Coma Scale (unit) & $-5.6(-5.7$ to -5.5$)$ & $0.3(0.2$ to 0.3$)$ & $-5.8(-5.9$ to $-5.7 ; p<0.001)$ \\
\hline Pulse (per minute) & 7.4 (6.6 to 8.2$)$ & $-4.5(-4.6$ to -4.4$)$ & $11.9(11.0$ to $12.7 ; p<0.001)$ \\
\hline
\end{tabular}

${ }^{a}$ All changes in vital signs calculated as the last minus the first measurement on scene. Means are adjusted for age, initial GCS, year, initial respiratory rate, initial pulse rate, initial systolic blood pressure and Elixhauser comorbidity score

\section{Definitions}

Strokes and TBI are defined by ICD10-AM codes and subtyped for ischemic and haemorrhagic strokes as well as TBI. Rapid sequence intubation is defined as the attempted or successful placement of an endotracheal tube in the trachea after receiving a paralytic agent. Successful placement of endotracheal tube in the trachea was confirmed using clinical means and end-tidal $\mathrm{CO}_{2}$ waveform.

\section{Statistical analysis}

Stata version 14 (Stata Corp, College Station, Texas, USA) was used to analyse data. Categorical variables are presented as frequencies, and continuous variables as means with standard deviations. Categorical variables were compared with the $\chi^{2}$ test and continuous predictors with the t-test or Wilcoxon rank-sum test. Hypothesis tests were two-sided, with a significance level of $p<$ 0.05 .

\section{Model building}

We utilized a maximum likelihood-fitted logistic regression model on complete observations only. Non-linear main-effect terms were fitted using fractional polynomials. Goodness of fit was tested using the Hosmer and Lemeshow test on a random sample, due to the tests excess sensitivity to large datasets $[11,12]$. Four regression models were assembled. The first was built on all non- missing observations of TBI and strokes to test interactions. Three additional models were constructed to calculate the effect of RSI for TBI, ischemic and haemorrhagic stroke separately. Previous work using this same dataset showed that missingness were clinically insignificant and did not meaningfully impact estimates [4]. As such, no sensitivity analyses for the impact of missing data were conducted.

\section{Interaction}

This analysis tested how the components of RSI interact with stokes and TBI for survival in the RSI-only group. Any such interactions in the RSI-only group might indicate that RSI influences survival differently for strokes compared to TBI, suggesting that the brain trauma RSI evidence cannot be applied to strokes. Rapid sequence intubation consists of many elements including medications, intubation success proportions and number of attempts, scene time and time to intubation. All these factors were tested for interaction and all interactions are presented graphically in this text.

Medications assessed for interaction with TBI and strokes include atropine, midazolam, midazolam/morphine infusions and fentanyl, as only these medications had sufficient data permitted an interaction test. Furthermore, RSI is likely to directly impact blood pressure, pulse rate, oxygen saturation, Glasgow Coma Scale, respiratory rate, end-tidal carbon dioxide [4]. Therefore, 


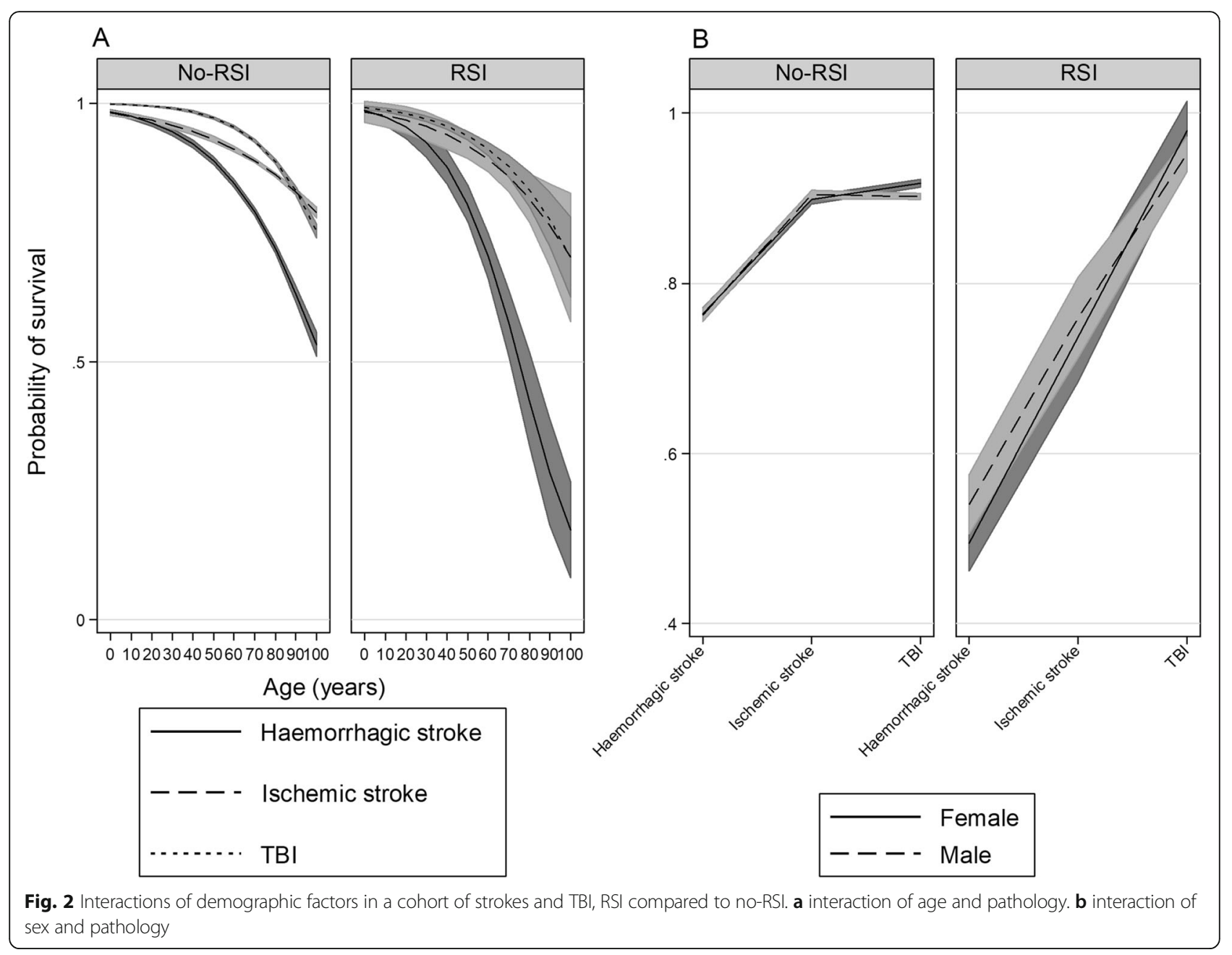

we also tested the interactions of vital signs. These vital sign changes were calculated by subtracting the final onscene value from the first on-scene measurement [4]. All interaction terms that were statistically significant at a 5 $\%$ level were considered as evidence of interaction. Results of interactions are presented in the manner suggested by Knol and van Der Weele [13], and we present the relative excess risk due to interaction in supplementary tables [14].

\section{Results}

This cohort included 107,128 (Fig. 1) of which 1993 (1.9\%) received RSI. A total of 80,324 (75\%) had complete data of which 11,133 (13.9\%) were diagnosed with haemorrhagic stroke and 26,754 (33.3\%) with ischemic stroke and $42,437(52.8 \%)$ with TBI. Baseline characteristics of the full-data cohort are compared in Table 1. The overall intubation success was $97.8 \%$ and first pass success was $89.6 \%$, with no significant change in success over time for overall $(p=0.40)$ or first-pass $(p=0.12)$.

\section{Overall outcomes}

After removing missing data, 80,324 strokes and TBI were transported by ambulance of which 71,008 (88.4\%) survived to hospital discharge. In an unadjusted regression those that had received RSI had 88\% lesser odds of survival; OR 0.09 (95\% CI 0.08 to $0.10 ; p<0.001$ ) compared to those patients that did not receive RSI. An adjusted regression analysis found no significant difference in survival for TBI, with $-0.7 \%$ lesser survival for RSI compared to no-RSI; OR 0.86 (95\% CI 0.67 to $1.11 ; p=0.25$ ) (Additional file 1: Table S1A). Survival for haemorrhagic stroke was $-14.1 \%$ less for RSI; OR 0.44 (95\% CI 0.33 to $0.58 ; p=0.01)$ and was $-4.3 \%$; OR 0.67 (95\% CI 0.49 to $0.91 ; p=0.01$ ) lesser for ischemic strokes (Additional file 1: Table S1B and C). Model fit and performance statistics indicate good fit (Additional file 1: Table S3).

Table 2 shows that the decline in systolic blood pressure is tenfold larger for haemorrhagic strokes compared to TBI. Oxygen saturation increases more when RSI is utilized, and the largest rise is for ischemic strokes. Respiratory rate does not change meaningfully in the 


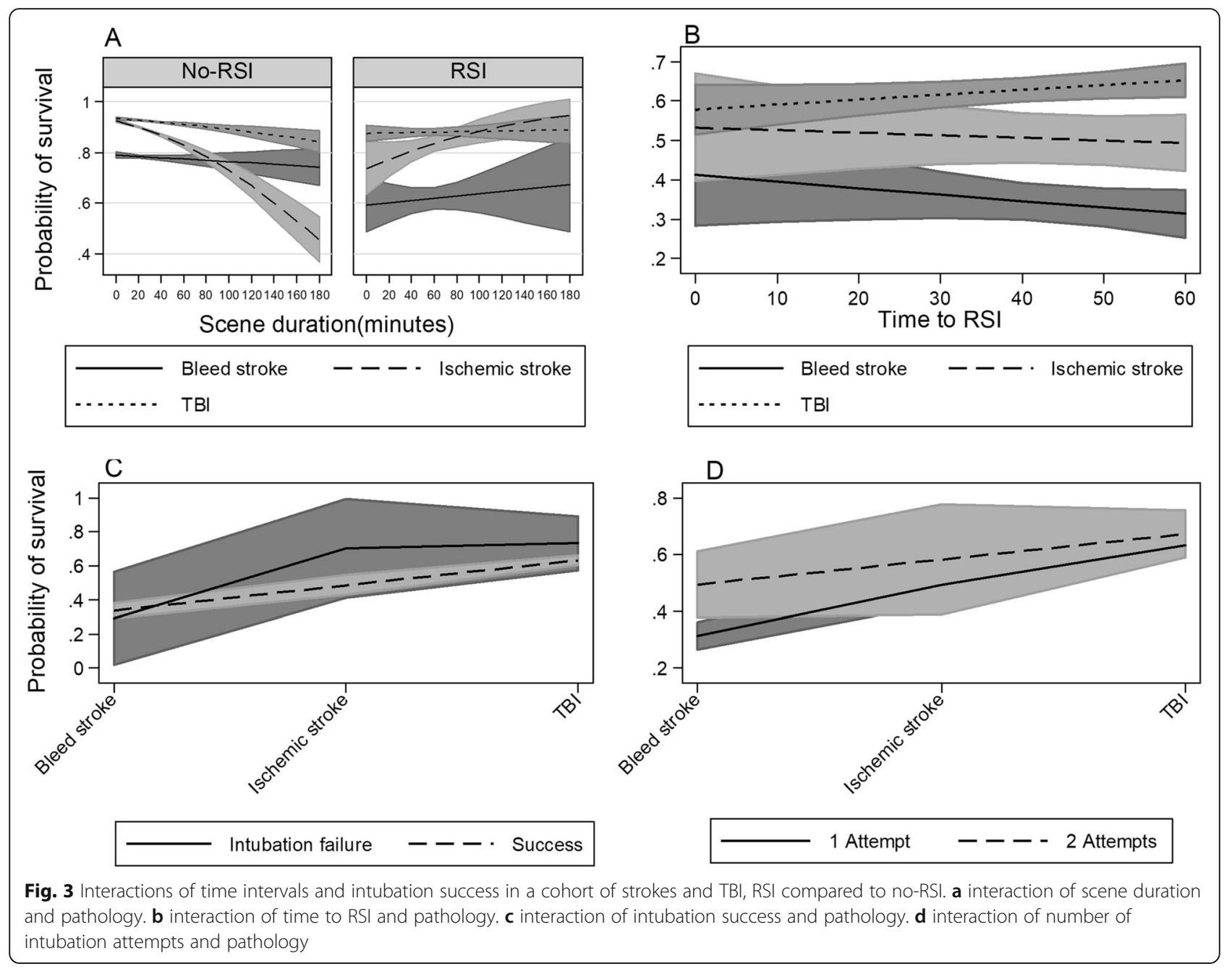

absence of RSI use, but there are six breaths per minute drop with RSI. Predictably, Glasgow Coma Scale decreases with RSI usage. For non-RSI patients pulse rate tends to decrease slightly on scene, but when RSI is utilized pulse rate increases, with haemorrhagic stroke double that of TBI and ischemic strokes.

\section{Interactions}

Here we report interactions that are statistically significant or borderline significant. All interactions are presented graphically in Figs. 2, 3, 4 and 5. For a complete report on all interactions, see Additional file 1: Table S2A to O.

\section{Demographic factors}

Age strongly predicted survival (Additional file 1: Table S1A to C). Age interacted with TBI and stroke in the RSI-only group $(p=0.02)$ (Additional file 1: Table S2A; Fig. 2a). A borderline insignificant interaction within the
RSI group exist when comparing sex $(p=0.06)$ (Additional file 1: Table S2B; Fig. 2b).

\section{Prehospital time intervals and intubation success}

No significant interaction for scene-time was found in the RSI-only setting $(p=0.18)$ (Additional file 1 : Table S2C; Fig. 3a). Even so, there was a borderline significant difference in the slopes for ischemic stroke vs TBI $(p=0.06)$. The mean time-to-RSI was $39.4 \mathrm{~min}(\mathrm{SD}=24.8)$, but there was no significant interaction for time-to-RSI $(p=0.10)$ (Additional file 1: Table S2D; Fig. 3b). However, a borderline significant difference between the survival slopes of TBI versus haemorrhagic strokes was noted $(p=0.05)$. We found no evidence of interaction of intubation success ( $p=0.49)$ (Additional file 1: Table S2E; Fig. 3c). Two or more attempts at intubation success was associated with increased survival for haemorrhagic strokes $(p=0.004)$, but not for ischemic strokes or TBI (Additional file 1: Table S2F; Fig. 3d). Nonetheless, both the number of 


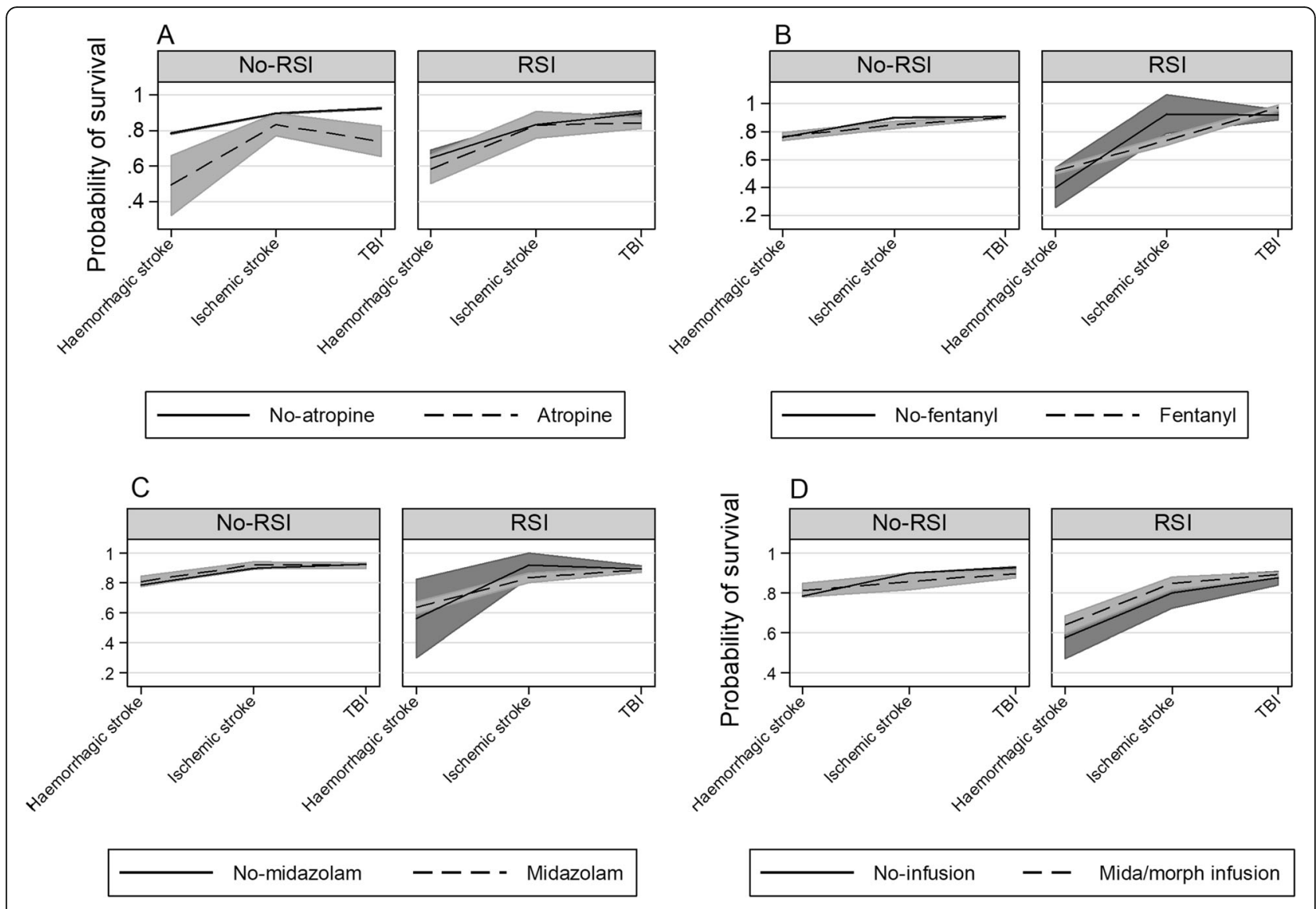

Fig. 4 Interactions of medications used with RSI in a cohort of strokes and TBI, RSI compared to no-RS. a interaction of atropine and pathology. $\mathbf{b}$ interaction of fentanyl and pathology. $\mathbf{c}$ interaction of midazolam and pathology. $\mathbf{d}$ interaction of midazolam/morphine infusion and pathology

intubation attempts and intubation success had low cell counts, limiting inferences from these two analyses.

\section{Rapid sequence intubation medications}

The interaction of atropine showed that survival was lower for TBI when atropine was used with RSI $(p=0.001)$ (Additional file 1: Table S2G; Fig. 4a). The interaction of fentanyl indicated that when fentanyl was used with RSI, survival is better for TBI $(p=0.01)$ (Additional file 1: Table S2H; Fig. $4 \mathrm{~b})$. No significant interactions for midazolam was apparent $(p=0.34)$ (Additional file 1: Table S2I; Fig. 4c), nor for midazolam/morphine infusions $(p=0.31)$ and $(p=0.84)$ (Additional file 1: Table S2J; Fig. 4d).

\section{Changes in vital signs after RSI use}

Survival decreased for haemorrhagic strokes and TBI as systolic blood pressure increased (Additional file 1: Table S1A to C). No interaction of systolic blood pressure for the RSI-only group is apparent $(p=0.61)$ (Additional file 1: Table S2K; Fig. 5a). No interaction was found for changes in respiratory rate $(p=0.52)$ (Additional file 1: Table S2L; Fig. 5b). For changes in $\mathrm{ETCO}_{2}$ with RSI use, no interaction is obvious $(p=0.91)$
(Additional file 1: Table S2M; Fig. 5c). No interaction for changes in $\mathrm{SPO}_{2}$ and TBI and stroke was found in the RSI-only setting $(p=0.91)$ (Additional file 1: Table S2N; Fig. 5d). For those that receive RSI significant differences exist between the stroke and TBI slopes of pulse rate change $(p=0.03)$ (Additional file 1: Table $\mathrm{S} 2 \mathrm{O})$; Fig. 5e).

\section{Discussion}

This analysis shows that RSI interact with strokes and traumatic brain injuries in terms of survival, indicating that the TBI evidence might not be useful to guide stroke RSI. As anticipated, this implies that the stroke patient differs from the TBI patient enough that RSI components impacts survival differently for these two pathologies. Significant interactions in the RSI-only group included number of intubation attempts, atropine, fentanyl, pulse rate and perhaps scene time, time to RSI as well as the age of the patient. Another suggestion that TBI evidence might not transferable is the difference in survival between the strokes and TBI for RSI. Haemorrhagic strokes have $14 \%$ lesser RSI survival, compared to 

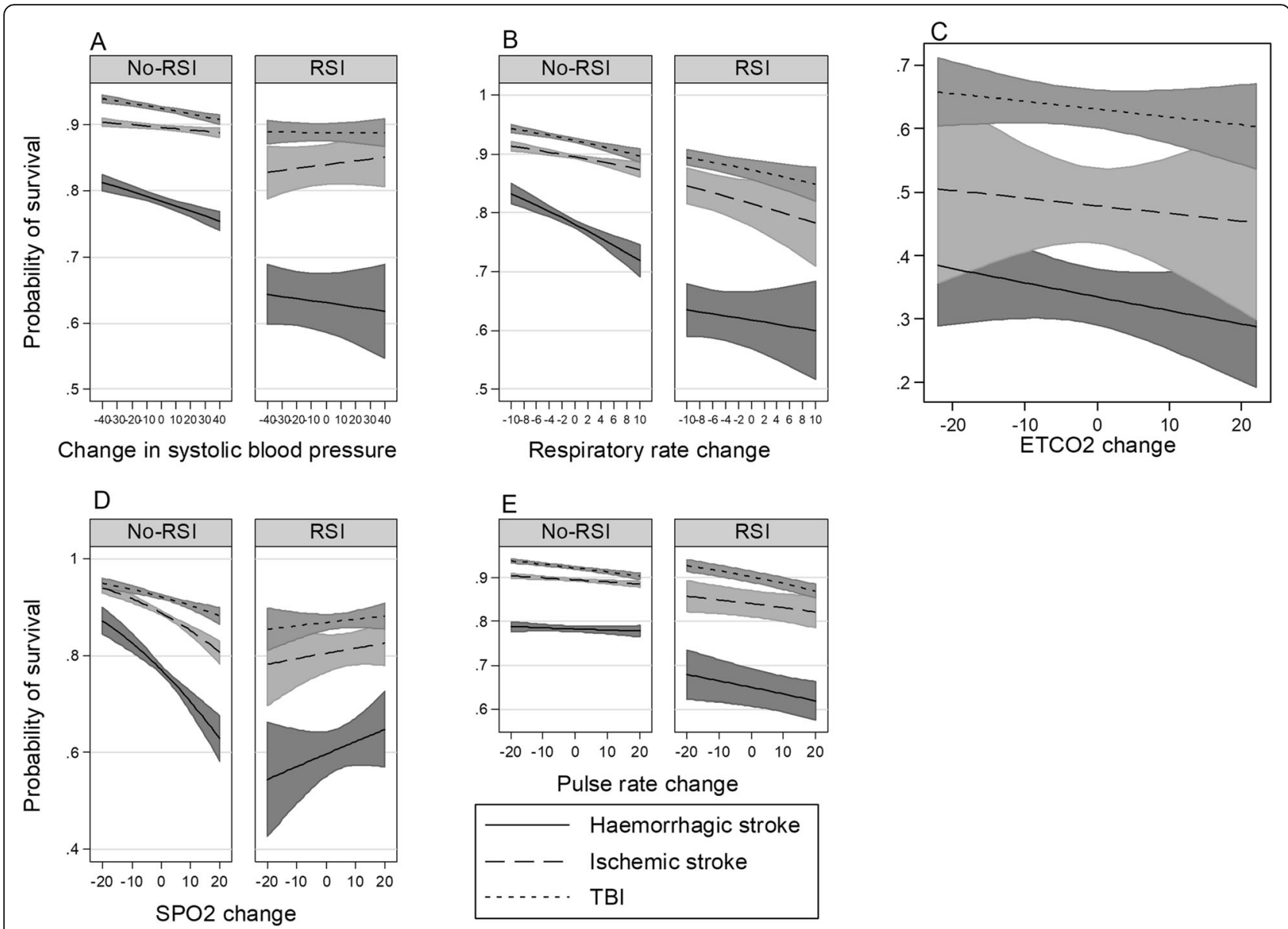

Fig. 5 Interactions of changes in vital signs in a cohort of strokes and TBI, RSI compared to no-RSI. a change in systolic blood pressure and pathology. $\mathbf{b}$ interaction of respiratory rate change and pathology. $\mathbf{c}$ interaction of ETCO2 change and pathology. $\mathbf{d}$ interaction of SPO2 change and pathology. e interaction pulse rate change and pathology

ischemic stroke of $4 \%$ and TBI a lesser than $1 \%$ survival difference between RSI and no-RSI.

Large differences in the changes in systolic blood pressure with RSI use are evident when comparing strokes and TBI. The largest decreases in RSI-related blood pressure were found in haemorrhagic strokes, and the least in TBI. Survival follows this pattern too, with haemorrhagic stroke the poorest survival compared to TBI the best. However, it is not clear that these decreases in blood pressure are the causes of poorer survival. Our analysis show that decreases in blood pressure with RSI was not associated with a significant decrease in survival. This finding of no decrease in survival after a decrease in blood pressure with RSI is mirrored in the results of the GOLIATH trial, which compared conscious sedation to general anaesthesia in ischemic stroke [15]. In the GOLIATH trial general anaesthesia caused a greater mean blood pressure drop compared to the conscious sedation group, similar to the drop in systolic blood pressure found with RSI in our analysis. Despite the decreased blood pressure caused by general anaesthesia in the GOLIATH trial, no decreased survival with such a blood pressure drop was found. Interestingly, survival seems to increase for haemorrhagic stroke after decreased blood pressure, but this effect was not statistically significant. This suggestion of increased survival with decreasing blood pressure after clinical interventions in haemorrhagic strokes have been found in other studies [16].

Age could be the most important driver of the differences in RSI survival between strokes and TBI, not blood pressure. Age decreases survival for both strokes and TBI, but the survival decrease is much more rapid for haemorrhagic strokes. These differences in the steepness of decline of the age slopes are important when one considers that almost three quarters of all stroke RSI in our analysis were haemorrhagic strokes, and the average age of these haemorrhagic stokes were 65 years, compared to TBI of 43 years. Given this large difference in age between TBI and haemorrhagic stroke for those that received RSI, and keeping in mind the much steeper decline with age in survival when comparing haemorrhagic stokes to TBI, the large difference between the survival for haemorrhagic stroke compared to TBI is unsurprising. Large decreases 
in survival with advanced age for patients with haemorrhagic strokes are consistent with another Australian study [17].

Other important interactions in the RSI-only group were evident. An increased number of intubation attempts were associated with increased survival for haemorrhagic strokes. This surprising and possibly confounded effect could result from less obtunded patients needing more intubation attempts. Another significant interaction was atropine, with lower survival for TBI when atropine was used. The mechanisms of this finding are unexplained. Furthermore, the interaction of fentanyl on the pathologies showed TBI have better survival when fentanyl is used and ischemic strokes worse survival with fentanyl use. Fentanyl is used to blunt the hemodynamic effects of intubation [18], but causes a decrease in mean arterial pressure [19]. it is possible that the blood pressure effects of fentanyl could be the cause of this medications impact on survival in this analysis. Furthermore, our analysis also found an interaction between pulse rate and pathology, with both strokes and TBI associated with decreased survival with increased pulse rate. We believe that the effect of pulse rate is mainly through the impact of blood pressure changes, and not pulse rate itself. Also, RSI use is associated with an apparent reversal of the deleterious effect of staying on scene longer. This could be due to potential benefits of RSI or perhaps a selection effect: patient that lived long enough to receive RSI at later scene times would cause an association of better survival with longer times on scene.

It is a strength of our study that our measures of prognostic risk due to illness severity and comorbidities together predicted survival well with an area under curve of 0.83 , which is higher than the 0.75 that would provide evidence of adequate risk adjustment [20]. Even so, it is possible that these results are still somewhat biased due to unmeasured confounding. Another strong point of our study is that it had a very large sample size, and consequently good statistical precision. This study has limitations. Firstly, the utility of observational methods to reveal treatment effects are limited, especially if the anticipated effect is small [21]. Secondly, we could not compare good neurological survival, which would have been a more suitable. Thirdly, we calculated the change in vital signs by subtracting the first values upon scene arrival from the last values before handover at the emergency department. Additionally, we believe that comparing the first vital signs measured upon arrival at the patient's side to the last vital signs (measured after all prehospital treatments were given) would show the impact of the effects of RSI on these vital signs after adjustment for confounders. Our data did not allow for a comparison of vial sign changes during RSI. We did not consider non-linear interaction terms due to restrictions in the sample sizes of the RSI group, although we anticipated non-linear effects for many variables.

\section{Conclusions}

Rapid sequence intubation and related factors interact with stroke and TBI, which implies that RSI effects stroke survival differently from TBI. If RSI impact survival differently for strokes compared to TBI, then perhaps the TBI evidence cannot be used for stroke RSI. A trial comparing RSI to no-RSI in the out-of-hospital setting is urgently needed.

\section{Supplementary information}

Supplementary information accompanies this paper at https://doi.org/10. 1186/s12873-020-0303-9.

Additional file 1: Table S1. A. Estimates of a logistic regression model of RSI adjusted for covariates in 42,437 traumatic brain injuries. B. Estimates of a logistic regression model of RSI adjusted for covariates in 29,457 ischemic strokes. C. Estimates of a logistic regression model of RSI adjusted for covariates in 14,374 haemorrhagic strokes. Table S2. Rapid sequence intubation effect modification of factors. Table S3. Model Fit and performance statistics.

\section{Abbreviations \\ GCS: Glasgow Coma Scale; RSI: Rapid Sequence intubation; TBI: Traumatic brain injury}

\section{Acknowledgements}

We are grateful to the staff at the Centre for Victorian Data Linkage as well as Salman Sabir at Ambulance Victoria for their assistance in obtaining the data for this study.

\section{Authors' contributions}

PFF conceived of the study. PFF, PAJ, MB, SB and KS all contributed to the methodological design of this research. KS and PFF collected data and PFF analysed all data. PFF, PAJ, MB, SB and KS interpreted the data and also contributed to the manuscript. All authors have read and approved the manuscript. PFF takes responsibility for the paper as a whole.

Funding

No funding for this study.

Availability of data and materials

Data is not available due to privacy concerns of patients.

Ethics approval and consent to participate

Monash University Human Research Ethics Committee provided ethics approval (ref. no. 8618).

Consent for publication

Not Applicable.

\section{Competing interests}

Co-author Paul Andrew Jennings is a member of the editorial board (Associate Editor) of BMC Emergency Medicine.

\section{Author details}

${ }^{1}$ Department of Community Emergency Health and Paramedic Practice, Monash University, Melbourne, Australia. ${ }^{2}$ Ambulance Victoria, Doncaster, Australia. ${ }^{3}$ School of Medicine, Griffith University, Gold Coast, Australia. ${ }^{4}$ Research and Evaluation, Ambulance Victoria, Blackburn North, Australia. ${ }^{5}$ Department of Epidemiology and Preventive Medicine, Monash University, Melbourne, Australia. 
Received: 19 November 2019 Accepted: 13 January 2020

Published online: 28 January 2020

\section{References}

1. Collaborators GBDCoD. Global, regional, and national age-sex specific mortality for 264 causes of death, 1980-2016: a systematic analysis for the global burden of disease study 2016. Lancet. 2017;390(10100):1151-210.

2. Fouche PF, Stein C, Jennings PA, Boyle M, Bernard S, Smith K. Review article: emergency endotracheal intubation in non-traumatic brain pathologies: a systematic review and meta-analysis. Emerg Med Australas. 2019;31(4):533-41.

3. Fouche PF, Jennings PA, Smith K, et al. Survival in out-of-hospital rapid sequence intubation of non-traumatic brain pathologies. Prehospital Emergency Care. 2017;21(6):700-8.

4. Fouche PF, Smith K, Jennings PA, Boyle M, Bernard S. The association of paramedic rapid sequence intubation and survival in out-of-hospital stroke. Emerg Med J. 2019;36(7):416-22.

5. Bernard SA, Nguyen $V$, Cameron $P$, et al. Prehospital rapid sequence intubation improves functional outcome for patients with severe traumatic brain injury: a randomized controlled trial. Ann Surg. 2010;252(6):959-65.

6. Ambulance Victoria. Clinical Practice Guidelines for Ambulance and MICA paramedics. Version 1.1 ed. Doncaster: Ambulance Victoria; 2016. p. 42-7.

7. Mansour O, Megahed MM, Abd Elghany EHS. Acute ischemic stroke prognostication, comparison between Glasgow Coma Score, NIHS Scale and Full Outline of UnResponsiveness Score in intensive care unit; 2014.

8. Lecky F, Woodford M, Edwards A, Bouamra O, Coats T. Trauma scoring systems and databases. Br J Anaesth. 2014;113(2):286-94.

9. van Walraven C, Austin PC, Jennings A, Quan H, Forster AJ. A modification of the Elixhauser comorbidity measures into a point system for hospital death using administrative data. Med Care. 2009;47(6):626-33.

10. Montgomery JM, Nyhan B, Torres M. How conditioning on posttreatment variables can ruin your experiment and what to do about it. Am J Polit Sci. 2018;62(3):760-75.

11. Hosmer DW, Lemeshow S, Sturdivant RX. Applied logistic regression. Wiley series in probability and statistics. 3rd ed. Hoboken: Wiley; 2013. p. 1. online resource ( $v$.) ill

12. Kramer AA, Zimmerman JE. Assessing the calibration of mortality benchmarks in critical care: the Hosmer-Lemeshow test revisited. Crit Care Med. 2007;35(9):2052-6.

13. Knol MJ, VanderWeele TJ. Recommendations for presenting analyses of effect modification and interaction. Int J Epidemiol. 2012:41(2):514-20.

14. Rothman KJ, Greenland S. Modern epidemiology. 2nd ed. Philadelphia: Lippincott-Raven; 1998.

15. Simonsen $\mathrm{CZ}$, Yoo AJ, Sorensen $\mathrm{LH}$, et al. Effect of general anesthesia and conscious sedation during endovascular therapy on infarct growth and clinical outcomes in acute ischemic stroke: a randomized clinical trial. JAMA Neurol. 2018;75(4):470-7.

16. Lattanzi S, Silvestrini M. Blood pressure in acute intra-cerebral hemorrhage. Ann Transl Med. 2016:4(16):320

17. Lee $A H$, Somerford PJ, Yau KK. Factors influencing survival after stroke in Western Australia. Med J Aust. 2003;179(6):289-93.

18. Jensen AG, Callesen T, Hagemo JS, et al. Scandinavian clinical practice guidelines on general anaesthesia for emergency situations. Acta Anaesthesiol Scand. 2010;54(8):922-50.

19. de Nadal M, Munar F, Poca MA, Sahuquillo J, Garnacho A, Rossello J. Cerebral hemodynamic effects of morphine and fentanyl in patients with severe head injury: absence of correlation to cerebral autoregulation. Anesthesiology. 2000;92(1):11-9.

20. Sjoding MW, Luo K, Miller MA, Iwashyna TJ. When do confounding by indication and inadequate risk adjustment bias critical care studies? A simulation study. Crit Care. 2015:19(1):195

21. Sherman RE, Anderson SA, Dal Pan GJ, et al. Real-world evidence - what is it and what can it tell us? N Engl J Med. 2016;375(23):2293-7.

\section{Publisher's Note}

Springer Nature remains neutral with regard to jurisdictional claims in published maps and institutional affiliations.

\section{Ready to submit your research? Choose BMC and benefit from:}

- fast, convenient online submission

- thorough peer review by experienced researchers in your field

- rapid publication on acceptance

- support for research data, including large and complex data types

- gold Open Access which fosters wider collaboration and increased citations

- maximum visibility for your research: over $100 \mathrm{M}$ website views per year

At $\mathrm{BMC}$, research is always in progress.

Learn more biomedcentral.com/submissions 